Commentary

\title{
The emerging role of human PYHIN proteins in innate immunity: Implications for health and disease
}

\author{
Dympna J. Connolly, Andrew G. Bowie* \\ School of Biochemistry and Immunology, Trinity Biomedical Sciences Institute, Trinity College Dublin, Dublin 2, Ireland
}

\section{A R T I C L E I N F O}

\section{Article history:}

Received 31 July 2014

Accepted 28 August 2014

Available online $\mathrm{xxx}$

\section{Keywords:}

Innate immunity

Pattern recognition receptors

DNA sensing

Signal transduction

Viral evasion

\begin{abstract}
A B S T R A C T
The innate immune response depends on the ability of immune cells to detect pathogens through germline-encoded pattern recognition receptors (PRRs). Recently discovered PRRs include some members of the Pyrin and HIN domain (PYHIN) family, which are encoded on an interferon-inducible gene cluster located on chromosome 1q23. There are five human PYHIN proteins; Absent in melanoma 2 (AIM2), IFN- $\gamma$ inducible protein 16 (IFI16), Myeloid cell nuclear differentiation antigen (MNDA), Pyrin and HIN domain family member 1 (PYHIN1) and the recently identified Pyrin domain only protein 3 (POP3). Early studies reported roles for these proteins in cell cycle control, tumour suppression and transcriptional regulation. AIM2 and IFI16 have now been shown to be immune sensors of non-self DNA, such as that produced by viruses in infected cells. AIM2 binds DNA to activate the inflammasome, while IFI16 detection of DNA can lead to the up-regulation of type I interferons or inflammasome activation. Recent studies have shown how IFI16 senses DNA viruses, and also how viruses evade detection by IFI16, while structural studies have greatly advanced our understanding of how AIM2 and IFI16 bind DNA to activate these immune responses. Furthermore, following the identification of POP3, interplay between members of this gene cluster has been established, with POP3 acting as a negative regulator of the AIM2 and IFI16 inflammasomes. In this review we discuss the current understanding of how PYHIN proteins function in innate immunity, their role in disease and the therapeutic possibilities that arise as a result.
\end{abstract} (c) 2014 Elsevier Inc. All rights reserved.

\section{Introduction}

The innate immune system is composed of germline-encoded pattern recognition receptors (PRRs) that collectively serve as sensors for monitoring the extracellular and intracellular compartments for signs of infection or tissue injury. These PRRs, which are responsible for the detection of pathogen associated molecular

Abbreviations: AIM2, absent in melanoma 2; ALR, AIM2-like receptor; ASC, apoptosis-associated speck-like protein containing a CARD; CARD, caspase activation and recruitment domain; cGAMP, cyclic GMP-AMP; cGAS, cGAMP synthase; DAI, DNA-dependent activator of IFN-regulatory factors; DAMP, damage associated molecular pattern; EBV, Epstein-Barr virus; HIN, hematopoietic interferon-inducible nuclear antigen; HIV-1, human immunodeficiency virus 1 ; HSV-1, herpes simplex virus 1 ; IFI16, IFN- $\gamma$ inducible protein 16 ; IFN, interferon; IL, interleukin; ISG, immune stimulated gene; KSHV, Kaposi's sarcoma-related herpes virus; MNDA, myeloid cell nuclear differentiation antigen; MTB, Mycobacterium tuberculosis; NLR, nucleotide oligomerisation domain-like receptor; PAMP, pathogen associated molecular pattern; PYD, pyrin; PYHIN, pyrin and HIN domain family member; POP3, pyrin domain only protein 3; PRR, pattern recognition receptor; RLR, retinoic acid inducible gene-like receptor; SLE, systemic lupus erythematosus; STING, stimulator of interferon genes; TLR, toll-like receptor; YY1, ying yang 1.

* Corresponding author. Tel.: +3531 8962435; fax: +35316772400.

E-mail address: agbowie@tcd.ie (A.G. Bowie). patterns (PAMPs) which signal the presence of a pathogen, and damage associated molecular patterns (DAMPs) which signalling tissue injury, include the Toll-like receptors (TLRs), the retinoic acid inducible gene-like receptors (RLRs) and the nucleotide oligomerisation domain-like receptors (NLRs) [1]. Recently the classification of a new group of PRRs has been proposed. These are the AIM2-like receptors (ALRs) that comprise some members of the PYHIN family, namely human AIM2 and IFI16 and murine p204 [2].

The ALRs are intracellular innate immune sensors responsible for the detection of DNA. As a pathogen-derived PAMP, DNA is capable of stimulating protective immunity to infection while the erroneous detection of self-DNA, where DNA likely acts as a DAMP, can provoke excess inflammation and autoimmunity. The human ALRs AIM2 and IFI16 are encoded on an interferon (IFN)-inducible gene cluster found on chromosome 1q23. This region also encodes three other PYHIN proteins; MNDA, PYHIN1 and POP3 [3-6]. While this region encodes five proteins in humans, other mammals have variable family expansions, with at least thirteen family members present in mice [7]. Collectively these proteins are referred to as PYRIN and HIN domain-containing (PYHIN) proteins since they possess an N-terminal PYRIN (PYD) domain and in most cases at least one C-terminal hematopoietic interferon-inducible nuclear 
antigen with 200 amino acid repeats (HIN-200 or HIN) domain (Fig. 1). The PYD domain (also known as DAPIN or PAAD domain) belongs to the death domain superfamily and is an alpha-helical motif that forms homotypic interactions with other PYD containing proteins. The 200 amino acid DNA binding HIN domain is classified into three subtypes, termed A, B and C, based on consensus motifs [8]. POP3, the most recent member of this gene cluster to be identified, lacks the HIN domains found in other PYHIN proteins [3].

The PYHIN proteins have been shown to localise to both the nucleus and cytosol. AIM2 and POP3 are predominantly cytosolic proteins, while IFI16, MNDA and PYHIN1 are predominantly found in the nucleus owing to the presence of an N-terminal nuclear localisation sequence (NLS) $[3,9]$. However, other factors such as acetylation or the presence of stimulatory DNA (in the case of IFI16), or the onset of apoptosis (in the case of MNDA), can also influence their cellular localisation [10-12].

Due to recent exciting discoveries demonstrating roles for some PYHIN proteins as innate immune PRRs, in this review we re-visit the functions of these proteins. Here we will focus on the human PYHINs, discussing earlier studies on this protein family, their new roles in DNA sensing and transcriptional regulation, the mechanisms by which these events occur and possible approaches to targeting PYHINs therapeutically.

\section{Early research on the human PYHINs}

Prior to discoveries demonstrating that members of the PYHIN family function in pathogen recognition, studies focused on roles for these proteins in cell growth, cell cycle control, cell differentiation, tumour suppression, apoptosis, and the DNA damage response.

MNDA, the first human PYHIN family member discovered, was identified in HL-60 cells as a $55 \mathrm{kDa}$ protein that is expressed predominantly in the nucleus and specifically in cells of the myeloid lineage [13]. Early studies into the role of MNDA suggested an involvement in myeloid differentiation. This idea was supported by studies revealing that MNDA can bind nucleolin and nucleophosmin $[14,15]$, both of which have been shown to be involved in the maturation and biosynthesis of ribosomes. The noted nuclear localisation, and lineage- and stage- specific expression of MNDA suggested that this PYHIN protein may play a role in regulating gene transcription. MNDA has been shown to bind to the transcription factor ying yang 1 (YY1), forming a ternary complex along with the YY1 target DNA. The interaction between MNDA and YY1 increases the affinity of YY1 for its target DNA and decreases its rate of dissociation [16]. This means that cellular levels of MNDA could have a dramatic effect on the transcription of several genes, highlighting its potential as a key regulator of gene expression. Indeed, retroviral mediated expression of MNDA in K592 cells, which normally lack MNDA, correlated with induction of delta-like 1 (DLK1), whose expression is essential for normal haematopoiesis, and specifically for myeloid cell development [17]. MNDA itself has also been proposed to act as a transcription factor in monocytes [18] and this is supported by a more recent study demonstrating that MNDA can directly bind double stranded DNA (dsDNA) [19]. A possible role for MNDA in neutrophil apoptosis has also been proposed. Thus during apoptosis, MNDA is cleaved by caspases and then relocalises to the cytosol where it accumulates and associates with the antiapoptotic protein myeloid cell leukaemia 1 (MCL1). This leads to MCL1 degradation, resulting in apoptosis caused by mitochondrial dysfunction [12]. This relocalisation of MNDA to the cytosol is thought to be required for proper execution of apoptosis and suggests that reduced cytoplasmic accumulation of MNDA contributes to suppression of neutrophil apoptosis. Furthermore, in septic patients cytoplasmic accumulation and cleavage of MNDA in neutrophils was dramatically impaired [12].

The second human PYHIN family member identified was IFI16. IFI16 migrates as three distinct protein species (IFI16A, B and C) on SDS-PAGE, the most abundantly expressed and commonly studied isoform being the $\mathrm{B}$ form. The three isoforms are generated as a

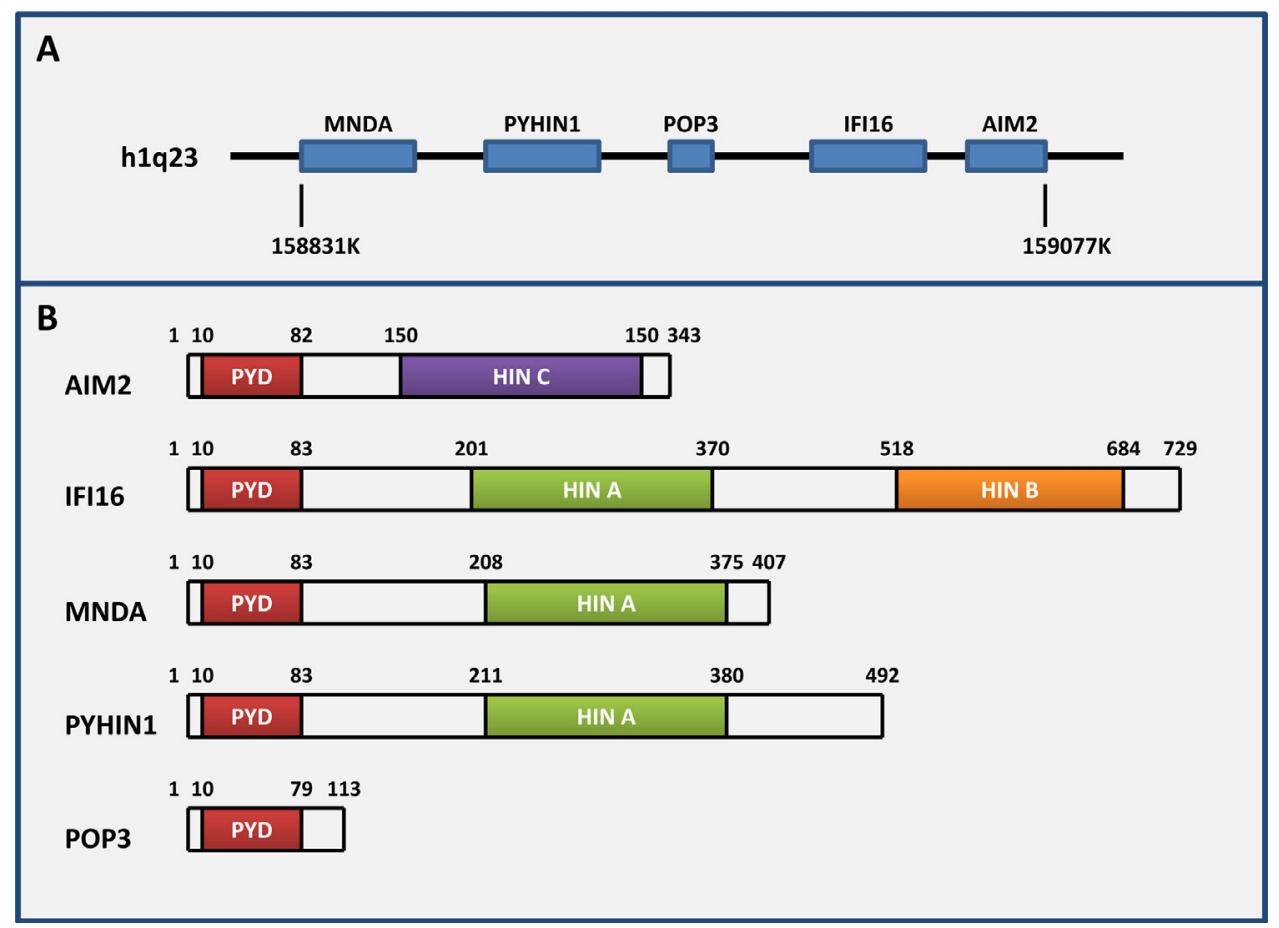

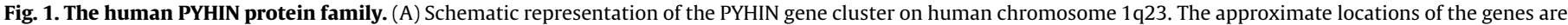

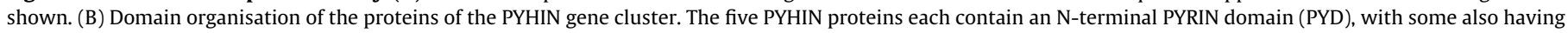
one or more C-terminal DNA binding HIN domains. These are classed as one of three subtypes; HIN A, HIN B or HIN C. 
result of alternative RNA splicing and are clustered at 85-95 kDa [20]. Early studies on IFI16 focused on its role in cell cycle control and transcriptional regulation. IFI16 has been shown to bind the Cterminal region of the tumour suppressor protein p53, augmenting p53-mediated transcriptional activation. While the mechanism of IFI16 modulation of p53 DNA binding is still not completely clear the data suggests that interaction of IFI16 with p53 may be important for binding of p53 to DNA, which then translates to an increase in the transcriptional activity of p53 [21]. This is reminiscent of MNDAs ability to enhance YY1 binding to DNA. Interestingly, IFI16 can also interact directly in vivo with the gene promoter region of both p53 and c-myc [22]. IFI16 has also been shown to bind BRCA1 and this complex has been reported to be involved in the p53-mediated cell death pathway, suggesting that IFI16 may play a role in DNA damage induced apoptosis [23]. However, IFI16 has also been shown to associate with the retinoblastoma tumour-suppressor protein $\mathrm{Rb}$ and the transcription factor E2F1, resulting in the inhibition of Rb-E2F1 mediated transcriptional repression [24]. While accumulating evidence supports the idea that PYHINs can act as transcriptional regulators, it highlights that this regulation can be indirect by influencing transcription factors, or direct resulting from a PYHIN:DNA association. By regulating transcription factors, IFI16 can also act as a restriction factor during human cytomegalovirus (HCMV) infection. It does so by interacting with the transcription factor Sp1, displacing it from promoters, thereby leading to a decrease in HCMV DNA synthesis, a decrease in viral gene expression and inhibition of viral replication [25]. Furthermore, IFI16 has recently been shown to be required for gene induction in response to innate immune RNA and DNA detection, since IFI16 is required for optimal RNA polymerase II occupancy on IFN- $\alpha$ and Immune Stimulated Gene (ISG) promoters [26]. This provides evidence for a role for IFI16 in transcriptional regulation and reveals a direct antiviral role for IFI16 to both DNA and RNA viruses. In addition, a prominent role for IFI16 in DNA sensing is now appreciated (see below).

Like IFI16, the third PYHIN protein identified namely AIM2 has a role in innate detection of DNA. However, prior to its identification as a DNA sensor, a role for AIM2 in tumour suppression was proposed. Indeed AIM2 was actually first identified in tumour cells lines whose tumorigenicity is intentionally suppressed [27]. Others supported this idea that AIM2 is a tumour suppressor and this role has been noted in many systems [28]. More recent work on AIM2 has focused on its role as a DNA sensor and will be discussed in more detail below.

The next PYHIN family member that was identified was PYHIN1 (or IFIX as it is also known). The PYHIN1 gene is predicted to encode six different protein isoforms as a result of alternative mRNA splicing ( $\alpha 1, \alpha 2, \beta 1, \beta 2, \gamma 1$, and $\gamma 2)$. Each of the isoforms has a common N-terminal region, which contains a PYD and a NLS. Consequently each of these proteins are localised in the nucleus. However, $\alpha 2, \beta 2$ and $\gamma 2$ have a deletion of nine amino acids in the $\mathrm{N}$-terminal region when compared to $\alpha 1, \beta 1$ and $\gamma 1$. In addition, isoforms $\alpha 1, \alpha 2, \beta 1$ and $\beta 2$ contain a HIN domain which is absent in both the $\gamma 1$, and $\gamma 2$ isoforms [6]. Like the PYD of other nuclear expressed PYHIN proteins, that of PYHIN1 also appears to form aggregates when expressed alone [29]. Furthermore, given that AIM2, IFI16 and MNDA have been shown to dimerise [15,20,30], this raises the possibility that their other closely related family member PYHIN1 can do so also. Functionally, roles for PYHIN1 in cell growth and tumour suppression have been reported. PYHIN1 expression has been reported to be reduced in breast tumours and breast cancer cell lines [6]. It is believed that PYHIN1 $\alpha 1$ possesses tumour suppressive activity mediated by its ability to interact with and destabilise the oncoprotein HDM2. This results in p53 stabilisation and activation of p53 responsive genes, such as the cyclin dependent kinase inhibitor p21CIP1. Consequently, CDK2 kinase activity is reduced and cell growth is inhibited [6,31]. A functional link between PYHIN1 $\alpha$ and maspin has also been proposed. Maspin is a serine protease inhibitor and suppressor of metastasis. In PYHIN1 $\alpha$ expressing cells, histone deacetylase 1 (HDAC1) is degraded, allowing maspin transcription which results in reduced metastasis [32].

The most recent addition to the PYHIN family is POP3 [3]. The gene encoding POP3 was recently shown to reside between PYHIN1 and IFI16 on the PYHIN locus (Fig. 1A). POP3 lacks a HIN domain responsible for DNA binding, but possesses a PYD domain. As such, POP3 can strongly interact with the ALRs AIM2 and IFI16, likely modulating their roles in innate immunity [3].

As yet, there is limited insight into the specific molecular roles of the PYHIN proteins in many of the diverse processes described above with which they have been associated. However, in recent years research has focused in particular on AIM2 and IFI16, given that an important role for these PYHINs in innate immunity has been uncovered. These significant discoveries will be now discussed in more detail, with a focus on the latest findings which have advanced our understanding of how cells sense the presence of pathogen and self DNA leading to activation of innate immunity.

\section{Intracellular DNA sensing}

The detection of intracellular DNA is a crucial event in the innate immune response to viruses and intracellular bacteria. However, aberrant DNA detection (for example of self DNA) can have negative consequences resulting in autoimmunity. While it has been appreciated for some years that DNA can be immunestimulatory, we have only recently begun to understand the molecular basis of DNA sensing. The endosomal TLR9 was the first described PRR for DNA [33]. However, following infections with intracellular pathogens, impairments in clearance of exogenous DNA or imbalanced control of endogenous DNA products, DNA can also accumulate in the cytosol. This leads to cytokine and IFN induction, especially if and when cytoplasmic DNases may be defective. Therefore, numerous studies attempting to identify cytosolic DNA sensors were undertaken. The first cytosolic DNA sensor identified was DNA-dependent activator of IFN-regulatory factors (DAI) [34]. However, as the sensing ability of DAI is restricted to specific cell types, additional DNA sensors were believed to exist. Indeed, to date more than ten cytosolic DNA sensors have been proposed (Fig. 2). Some of these DNA sensors can also function in the nucleus. While the other DNA sensors have been reviewed elsewhere [35,36], AIM2 and IFI16 as members of the PYHIN family are of particular interest here.

\section{Discovery of AIM2 as a DNA receptor}

While it has been known for some time that the HIN domain is a DNA binding domain present in the PYHIN proteins [37], it was some time before a PYHIN family member, namely AIM2, was implicated in host immune defence as a DNA sensor [9]. Unlike the other PYHINs it was revealed that AIM2 is predominantly cytosolic and can recognise non-self dsDNA in this compartment via its HIN domain [9]. Following dsDNA binding, the PYD and Caspase activation and recruitment domain (CARD) containing bipartite adapter protein ASC (apoptosis-associated speck-like protein containing a (ARD) is recruited to AIM2 via homotypic PYD:PYD domain interactions [9]. ASC recruitment allows for the formation of a large multi-protein inflammasome complex, which mediates caspase- 1 activation and interleukin-1 $\beta$ (IL-1 $\beta$ ) processing [9]. Given that human and mouse AIM2 are direct orthologues, studies investigating the role of AIM2 in DNA recognition and IL-1 $\beta$ production using AIM2 knockout mice have provided greater 


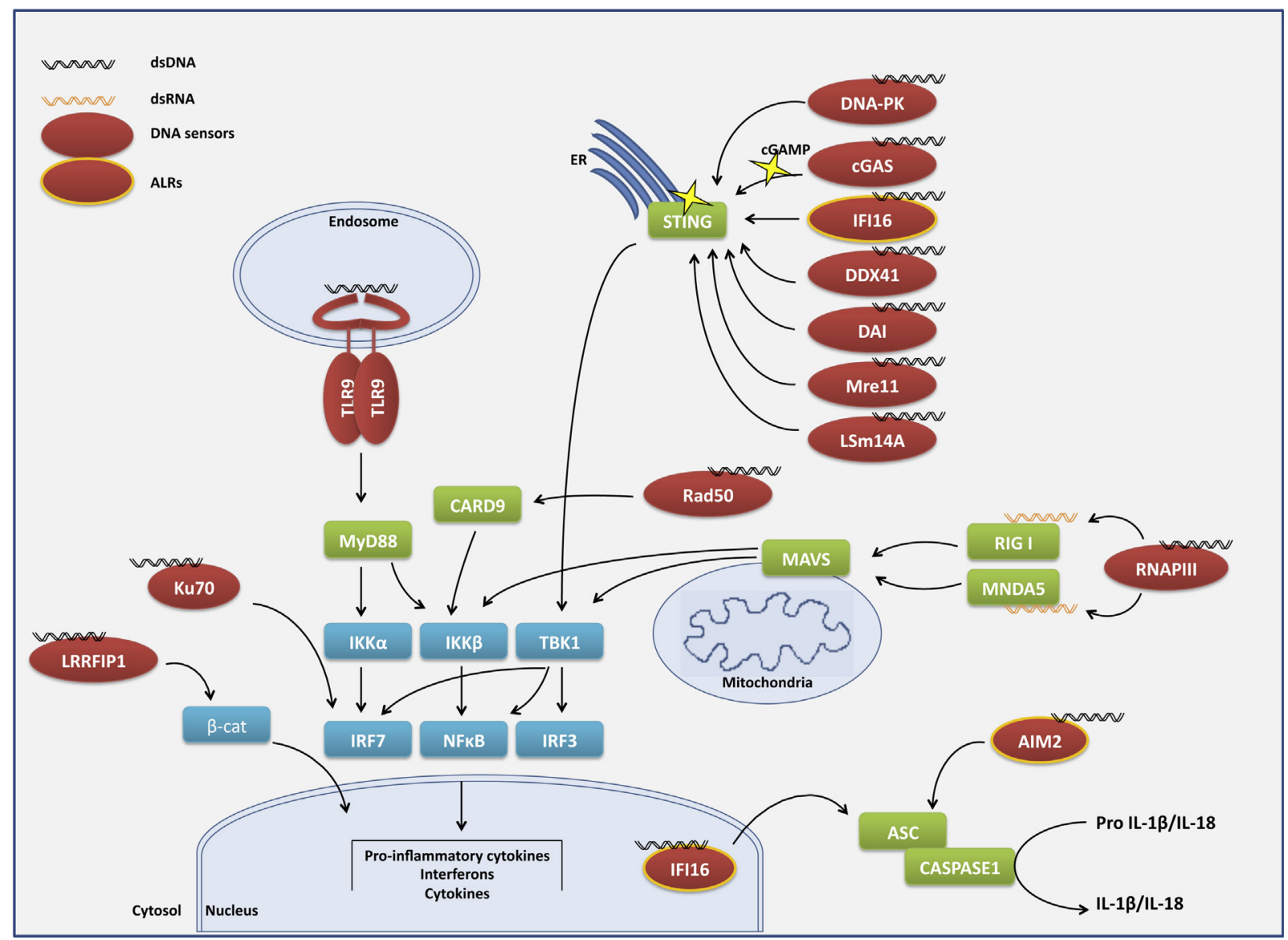

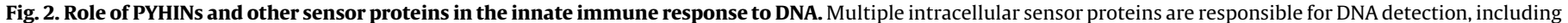

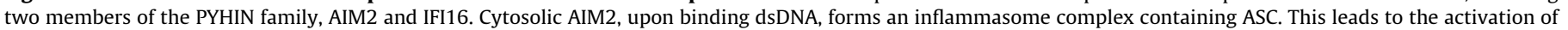

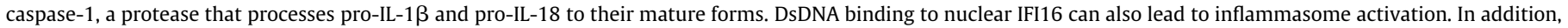

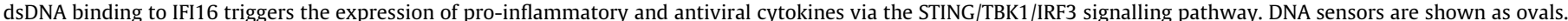

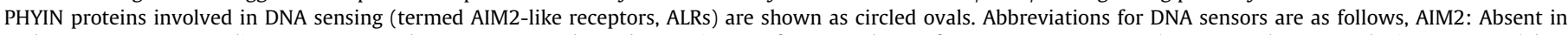

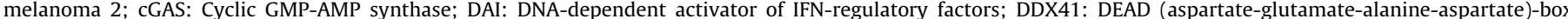

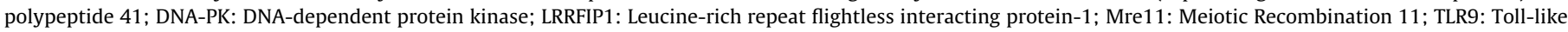
receptor 9; RNAPIII: RNA polymerase III.

insight into the role of AIM2 as a DNA sensor. While validating its role as a DNA sensor these studies have also demonstrated that AIM2 is involved in the detection of the cytosolic bacteria Francisella tularensis, vaccinia virus and Listeria monocytogenes [38], pathogens which can also infect humans. Although the involvement of AIM2 in Mycobacterium tuberculosis (MTB) detection in human remains to be investigated, AIM2 knockout mice are also highly susceptible to MTB demonstrating defective caspase-1 activation, IL-1 $\beta$ and IL-18 production [39]. Furthermore, mouse p204, the functional homologue of IFI16 contributes to IFN- $\beta$ and IFIT1 induction following MTB infection [40], suggesting that ALRs may also be responsible for MTB detection in humans.

More recent work on AIM2 has sought to investigate the structure of the HIN domain in complex with dsDNA and the molecular mechanisms involved in AIM2 inflammasome activation following DNA binding. The crystal structure of the dsDNA bound AIM2 HIN domain has revealed that AIM2 recognises DNA in a nonsequence specific manner via electrostatic attraction between positively charged HIN domain residues and the dsDNA sugarphosphate backbone [41]. Importantly, these findings provide a rationale for the permissive nature of DNA binding by AIM2. Docking and binding studies have revealed that AIM2 can selfassociate prior to dsDNA binding, via an AIM2HIN:AIM2PYD association [42]. This association maintains AIM2 in an autoinhibited state prior to dsDNA binding. Interestingly the negatively charged surface of the AIM2PYD which can associate with the HIN domain of AIM2 is the same region which binds the PYD domain of ASC following dsDNA binding. Such elegant mutually exclusive interactions are thought to ensure that AIM2 interacts with the downstream adapter ASC only upon activation of AIM2 by the dsDNA ligand. In addition to releasing AIM2 from its auto-inhibited state, dsDNA also acts as a platform for the recruitment of multiple AIM2 molecules, allowing for the close approximation of individual AIM2PYDs. These AIM2 oligomers in turn facilitate the nucleation of multiple ASCPYDs leading to the formation of the multi-protein inflammasome complex required for IL-1 $\beta$ processing [43].

\section{Viral DNA sensing by IFI16 leading to downstream signalling}

IFI16 is the second member of the human PYHIN family shown to be an innate immune sensor for dsDNA. Unlike AIM2, IFI16 is most abundant in the nucleus as a result of a NLS present in its Nterminal region [10]. IFI16 was initially identified as a novel DNA sensor using a 70 base pair IFN- $\beta$-inducing vaccinia virus dsDNA motif to purify DNA-binding proteins from cytosolic human monocytic extracts [2]. Unlike the DNA sensor TLR9, DNA sensing by IFI16 is independent of GC content and sequence [2]. As with AIM2, the sequence independent DNA binding by IFI16 is achieved by electrostatic attraction between positively charged HIN domain residues and the dsDNA sugar phosphate backbone [41]. This 
allows IFI16 to detect dsDNA from many sources including Kaposi's sarcoma-related herpesvirus (KSHV) [11], Epstein-Barr virus (EBV) [44], herpes simplex virus 1 (HSV-1) [45] and human immunodeficiency virus (HIV-1) [46]. On the contrary, dsDNA sensing by IFI16 is length dependent [2], with dsDNA fragments of 150 base pairs reportedly being favourable for the formation of an optimal binding cluster of approximately ten IFI16 protomers [47]. In order to bind stimulatory dsDNA, the HIN domains of IFI16 associate with both DNA strands across both the major and minor strands, in keeping with the requirement of dsDNA rather than ssDNA for the initiation of signalling from IFI16 [2,41]. While both HIN domains of IFI16 can associate with dsDNA, the HINb domain possesses a stronger DNA binding affinity $[2,41]$. In contrast to AIM2, DNA recognition by IFI16 can occur in both the cytosolic and nuclear compartments. For example DNA which has been transfected, lentiviral DNA that has accumulated or herpesvirus DNA which has been exposed in the cytosol can be detected by cytosolic IFI $16[2,46,48]$ while DNA from nuclear viruses such as HCMV is detected in the nucleus [10,49]. As mentioned earlier, the NLS of IFI16 is required for the nuclear localisation of IFI16. Recently, two acetylation sites that negatively regulate NLS function have been identified. When these lysine residues at position 99 and 128, which are within two critical NLS motifs are acetylated, nuclear import is inhibited (Fig. 3). The acetylation status of IFI16 is thought to be regulated by the acetyltransferase p300 and the histone deacetylase HDAC and occurs in various cell types [10]. Thus an important factor in the regulation of IFI16 localisation is its acetylation status.

Whether host receptors such as IFI16 have any capacity to distinguish self DNA from non-self DNA at the molecular level has been a long-standing question in innate immunity. An early dogma in the DNA sensing field was the idea that the nucleus was an immune-privileged cellular compartment for DNA detection. However, we now know that some sensors such as IFI16 can specifically detect non-host DNA in the nucleus. A recent paper examining IFI16 oligomerisation on DNA has provided insights into how this distinction might occur. Firstly, Morrone et al. found that following DNA binding, IFI16 continues to assemble on the dsDNA strand in a cooperative manner via PYD:PYD associations, forming IFI16 foci from which downstream signal activation can be initiated [47]. For optimal polymerisation to occur a region of approximately $150 \mathrm{bp}$ of DNA is required. Thus, despite exceeding the footprint of the HIN domains, the length of the exposed linkerdsDNA between nucleosomes (10-20 bp) or even that of the transcription bubble (approximately 17 bases) is too short to promote robust cooperative filament assembly of IFI16. This provides an elegant explanation for how the distinction is made been self and non-self DNA and identifies long naked DNA as the true IFI16 ligand. Secondly Morrone et al. revealed an important role for the IFI16 PYD domain, demonstrating that it is crucial for IFI16 oligomerisation.

Following the activation of IFI16 by immune stimulatory nonself DNA, this receptor recruits the endoplasmic resident protein STING (stimulator of interferon genes) [2]. The IFI16:STING association occurs in the cytosol following either cytosolic or nuclear DNA detection, as after herpesvirus DNA detection by nuclear IFI16 this portion of activated IFI16 translocates to the cytosol $[11,49]$. How exactly nuclear export of IFI16 is regulated after DNA sensing remains to be determined, but may involve

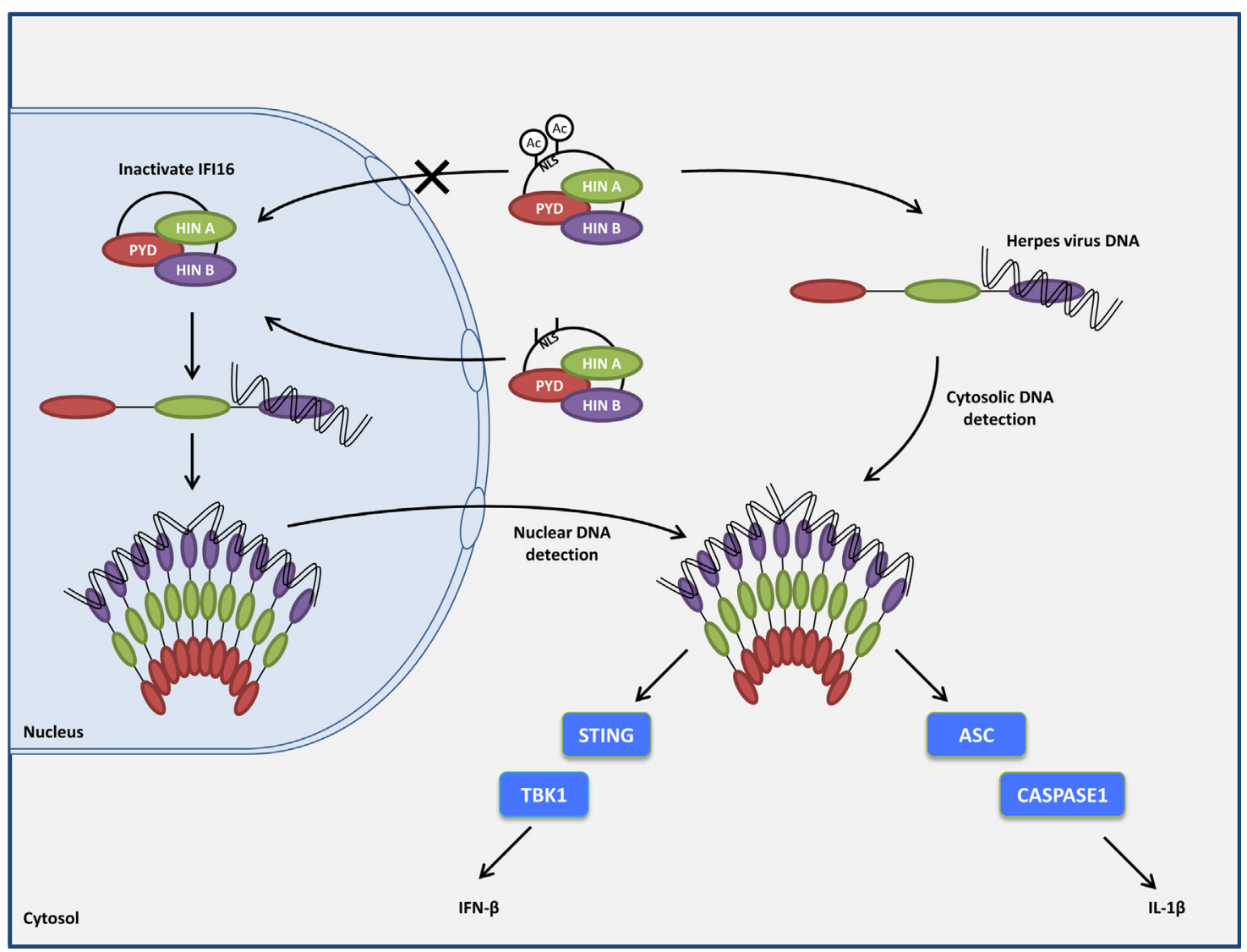

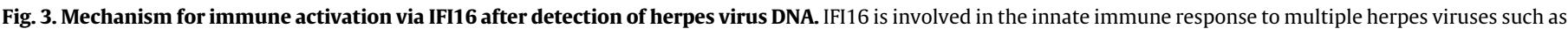

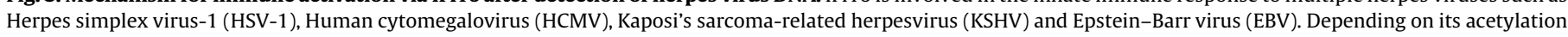

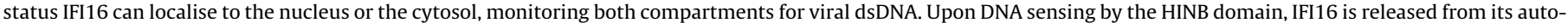

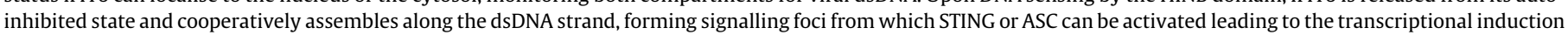
of type I interferon genes or proIL-1 $\beta$ processing respectively. 
regulated alteration of the acetylation status of IFI16, since the acetylation status of IFI16 regulates its cellular location (as described above). Alternatively, in cells such as macrophages, which are less permissive to viral infection, proteasomal degradation of viral capsids allows the release of viral DNA into the cytosol prior to nuclear delivery of the genome. This exposure of viral DNA activates cytosolic IFI16, which also leads to STING/TBK1 activation and IFN- $\beta$ expression [48]. While STING and TBK1 are widely accepted to be activated following IFI16 stimulation, the details of the molecular events involved in their activation have yet to be unravelled. STING is known to be activated upon binding of cyclic GMP-AMP (cGAMP) which is a novel second messenger produced by cGAMP synthase (cGAS). Like IFI16, cGAS has been shown to directly bind dsDNA, and in this case this binding event stimulates the catalytic activity of cGAS, leading to cGAMP production (Fig. 2). How IFI16 'activates' STING, and whether this involves cGAS and cGAMP is currently unclear, although we do know that both IFI16 and cGAS can contribute to DNA sensing in certain cell types [50].

Recently, IFI16 has been identified as a host receptor responsible for the detection of HIV DNA in macrophages and $\mathrm{CD}^{+} \mathrm{T}$ cells [46,51]. Importantly, in macrophages IFI16 was shown to bind not only dsDNA, but also ssDNA containing long stem structures and terminal loop regions that form a duplex structure, both which are produced during the HIV lentiviral life cycle [46]. As such, in macrophages IFI16 contributes to the detection and early control of HIV in these cells. However, abortive infection of $\mathrm{CD}^{+} \mathrm{T}$ cells with HIV, which leads to accumulation of HIV DNA, causes IFI16dependent pyroptosis [51], since like AIM2, IFI16 activation can also promote inflammasome activation in some contexts. IFI16-dependent inflammasome activation has been reported to also occur after infection with herpes viruses such as KSHV, HSV-1 and EBV $[11,44,45]$. However, HSV-1 and HCMV infection can also result in IFI16 dependent IFN- $\beta$ induction via the STING/TBK1 pathway. As yet the molecular details of the differential events involved in IFI16 dependent ASC and STING activation remain unclear.

Recent structural studies on DNA binding and oligomerisation as a key step in pathway activation for both AIM2 and IFI16 have provided some important answers to previously unresolved questions in the field, allowing us a more complete understanding of how these DNA sensors work. However, there is undoubtedly a great deal more to decipher, with many questions still remaining. For example, the exact cellular location of IFI16 induced STING activation remains elusive. It is also unclear if IFI16 polymerisation facilitates inflammasome formation as well as STING activation. In addition, while the importance of DNA length for IFI16 binding has been explored the correlation between optimal binding and downstream pathway activation has not. An analysis of the optimal ligand for AIM2 binding and inflammasome formation would also be very interesting.

\section{Host and pathogen regulation of AIM2 and IFI16}

Since AIM2 and IFI16 can drive inflammation via cytokine induction, and are also critical in early viral detection as PRRs, it is to be expected that there are numerous host and pathogen mechanisms to regulate them. Some of these mechanisms have evolved in humans, while others have co-evolved in pathogens to counter ALR functions in an attempt to evade detection, or to minimise the extent of the immune response.

An interesting mechanism of PYHIN regulation has recently been uncovered with the discovery of the newest member of the PYHIN family, POP3 [3]. POP3 lacks the HIN domain found in other PYHINs (Fig. 1), and so lacks any DNA binding ability. However, POP3 can directly bind both AIM2 and IFI16 via its PYD domain, and in doing so regulate downstream pathway activation. The association of POP3 with AIM2 diminishes the ability of AIM2 to associate with ASC thereby preventing inflammasome formation. Another example of co-regulation within the PYHIN family is AIM2 regulation by IFI16, where IFI16 can bind AIM2 and inhibit AIM2 mediated caspase- 1 activation [5], possibly by reducing the levels of free AIM2 available for ASC association. In mice, co-regulation within the PYHIN family has also been reported. Here, another PYHIN family member p202 is capable of inhibiting AIM2 [52]. p202 lacks a PYD but possess two DNA binding HIN domains, the first of which is capable of sequestering cytosolic dsDNA. Interestingly, the second HIN domain of $\mathrm{p} 202$ binds the HIN domain of AIM2, resulting in spatial separation of AIM2 PYD and the prevention of ASC clustering [53]. While no known human PYHIN possesses a similar domain architecture to mouse p202, alternatively spliced isoforms of human AIM2 have been predicted [54]. Perhaps those which lack either the PYD or the HIN domain can attenuate AIM2 activation in a manner similar to p202 inhibition of mouse AIM2. Finally, as mentioned earlier prior to dsDNA binding AIM2 is found in an autoinhibited state, where the PYD is in association with the HIN domain. Only following dsDNA binding is the AIM2PYD liberated and available for ASCPYD binding [42], providing another example of regulation within the PYHIN family itself.

As a result of co-evolution with their hosts, viruses have also acquired mechanisms for blocking host immune signalling. The first report of viral targeting of a PYHIN protein came from herpes simplex virus 1 (HSV-1). It had been known for some time that the HSV-1 immediate early protein ICPO was capable of inhibiting IRF3 signalling, however its mechanism of inhibition remained elusive for some time. The ability of ICP0 to target IFI16 for proteasomal degradation was proposed to be at least one key mechanism employed by ICPO for immune evasion [55]. Expanding on this work Johnson et al. demonstrate that ICP0 co-localises with IFI16 two and four hours post HSV-1 infection. In addition, this study demonstrated that IFI16 is phosphorylated following HSV-1 infection, likely targeting it for proteasomal degradation [45]. However, another study which raised concerns about the difficulties in distinguishing between the direct and indirect effects of ICP0, reasoned that ICPO does have an impact on IFI16 related activities but that IFI16 is not necessarily an ICP0 substrate [56]. Nevertheless, this study agrees that following HSV-1 infection IFI16 is degraded by the proteasome, which is a very effective mechanism to limit prolonged viral detection. MNDA may also be subject to targeting by viral proteins, as Latency-associated nuclear antigen (LANA) from KSHV has been shown to associate with MNDA, however the implications of this interaction are unknown [57].

It is becoming more apparent that innate host sensors frequently utilise aggregation dependent amplification mechanisms for signal activation $[43,58]$. Indeed, the HCMV protein pUL83 has been shown to interact with the PYD of IFI16, blocking its oligomerisation [29], and thus antagonising a critical step required for receptor activation. Utilising a HCMV strain lacking pUL83, Li et al. demonstrated that IFI16 oligomerisation is required for phosphorylation and nuclear translocation of IRF1 and IRF3. However, as no difference in caspase1 cleavage was noted, the importance of IFI16 oligomerisation for inflammasome activation remains unclear. Interestingly, amino acids in the IFI16PYD which are critical for IFI16 oligomerisation are not well conserved in AIM2 [47], raising the possibility that AIM2PYD either oligomerises differently or not at all, although overexpression of the AIM2PYD does result in filament formation [29]. Since AIM2 can nucleate ASCPYD, leading to the polymerisation of ASC in the AIM2 inflammasome [43,58], it remains a possibility that AIM2PYDs which are in very close proximity to each other rather than polymerised per se may be sufficient to nucleate ASC for inflammasome formation.

To date there have not been any extensive studies on viral inhibitors of PYHIN1 or MNDA. However, pUL83 binding of PYHIN1, as well as MDNA has also been reported [29]. Furthermore, 
co-expression of pUL83 with the PYD of MNDA or PYHIN1 also disrupted their ability to form nuclear oligomers. This raises the possibility that this HCMV protein is capable of interfering with the function of all three nuclear PYHIN proteins.

\section{Disease related roles of the PYHIN proteins}

While the current known roles of PYHIN proteins have placed them at the forefront of innate immunity, working as protectors of the host by sensing dsDNA from invading pathogens, there is now ample evidence showing that dysregulation of these pathways can have a negative impact on the host. For example, dysregulated inflammasome activity resulting in excessive IL- $1 \beta$ production, or inappropriate recognition of self nucleic acids leading to IFN- $\beta$ induction, can both potently promote particular autoimmune diseases.

Much of the evidence for roles for the PYHINs in disease comes from the analysis of their expression in various conditions. As can be seen in Table 1 dysregulated PYHIN expression can occur in many different conditions ranging from viral infections to cancer. However, in many cases while the association is clear it may be difficult to determine if these changes in expression patterns are a cause or effect of the disease in question, particularly due to the fact that many PHYIN proteins are IFN-inducible.

Genetic factors are also thought to be important in determining disease risk, for example, PYHIN proteins have been reported to lie within a susceptibility locus for systemic lupus erythematosus (SLE) [59] and the PYHIN1 locus has been identified as a novel susceptibility locus for asthma, specifically for individuals of African descent [60].

A protective role for AIM2 has been proposed in prostate diseases, with increased steady-state levels of AIM2 mRNA and protein in benign prostate hyperplasia but significantly lower levels of AIM2 expression in prostate cancer cells as compared to normal prostate epithelial cells [61]. Furthermore, reduced AIM2 expression in colorectal cancer is reportedly associated with poor outcome, suggesting a protective role for AIM2 here also [62]. In contrast elevated AIM2 levels and inflammasome activity have been reported in psoriatic lesions, possibly provoking this chronic autoimmune disease via sensing of self DNA [63]. Altered PYHIN1 and MNDA levels in a number of conditions have also been reported (Table 1 ).

IFI16 is a target for autoantibodies and several studies have shown the presence of anti-IFI16 autoantibodies in chronic autoimmune diseases such as SLE [64], systemic sclerosis [65], rheumatoid arthritis [66] and Sjögren's syndrome [67]. IFI16 is known to relocalise from the nucleus to the cytosol following UV treatment or viral DNA detection. It is hypothesised that IFI16 can also be released into the extracellular milieu. This extracellular IFI16 can in turn bind to the plasma membrane of endothelial cells, and down regulate both their migratory activities and tube morphogenesis [66]. In this context IFI16 may be acting as an alarmin, propagating the stress signal and causing further damage.

As mentioned earlier IFI16 is an important sensor for HIV DNA in multiple cell types [46,51]. This work was further supported with findings from HIV patients demonstrating that they have elevated IFI16 levels, possibly arising as a result of chronic immune activation. While elevated IFI16 levels did correlate with a high viral load and low $\mathrm{CD}^{+}$cell counts, IFI16 mRNA rather than protein levels were analysed [68]. This may not reflect protein expression levels of IFI16 and also does not take into account other factors such as subcellular localisation and protein trafficking. This limitation is also mirrored in some other studies and highlights the importance of further studies relating to the exact roles of PYHINs in disease.

While many studies are suggestive of a role of the human PYHINs in a number of conditions, mechanistic studies implicating these proteins in the pathogenesis of these diseases are still lacking and will need to be carried out if the PYHIN proteins are to be utilised as bio-markers or considered as therapeutic targets. Also, increased PYHIN expression during disease could be a consequence of increased IFN expression, present as a result of the disease rather than being a causative factor in the onset of the disease itself. However if this is the case, elevated PYHIN protein levels or the presence of anti-PYHIN antibodies may still be useful as biomarkers in certain diseases.

Table 1

Expression of PYHINs in disease.

\begin{tabular}{|c|c|c|c|}
\hline Protein & Disease & Comment & Reference \\
\hline \multirow[t]{6}{*}{ AIM2 } & $\begin{array}{l}\text { Abdominal aortic } \\
\text { aneurysm (AAA) }\end{array}$ & $\begin{array}{l}\text { Increased AIM2 protein expression in both sporadic infiltrating lymphoid cells and lymphoid } \\
\text { aggregates located in the outer media and adventitia of blood vessels }\end{array}$ & [76] \\
\hline & Colorectal cancer & Reduced expression of AIM2 protein associated with poor outcome & {$[62]$} \\
\hline & Hepatitis B virus (HBV) & Higher AIM2 protein expression in HBV associated glomerulonephritis tissue & [77] \\
\hline & Prostate cancer & Reduced AIM2 mRNA expression in tumour specimens & [61] \\
\hline & Psoriasis & Increased amounts of AIM2 mRNA and protein in lesional skin & [63] \\
\hline & $\begin{array}{l}\text { Systemic lupus } \\
\text { erythematosus (SLE) }\end{array}$ & $\begin{array}{l}\text { Increased AIM2 mRNA expression in SLE patients as a result of the reduced DNA methylation status of } \\
\text { the AIM2 gene }\end{array}$ & [78] \\
\hline \multirow[t]{7}{*}{ IFI16 } & HIV & Elevated IFI16 mRNA levels in peripheral blood mononuclear cells from untreated HIV patients & [68] \\
\hline & Rheumatoid arthritis (RA) & Autoantibodies against IFI16 present in sera of patients & {$[67,79]$} \\
\hline & Sjögren's syndrome (SjS) & Autoantibodies against IFI16 present in sera of patients & [66] \\
\hline & Systemic lupus & Autoantibodies against IFI16 present in sera patients & {$[64,79]$} \\
\hline & erythematosus (SLE) & $\begin{array}{l}\text { Increased IFI16 protein expression in all epidermal layers and in the dermal inflammatory infiltrates of } \\
\text { lesional skin }\end{array}$ & \\
\hline & Systemic sclerosis (SSc) & Autoantibodies against IFI16 present in sera of patients & {$[65,79]$} \\
\hline & & $\begin{array}{l}\text { Increased IFI } 16 \text { protein expression in all epidermal layers and in the dermal inflammatory infiltrates of } \\
\text { lesional skin }\end{array}$ & \\
\hline \multirow[t]{4}{*}{ MNDA } & Lymphoma & High MNDA protein expression in marginal zone lymphomas & [80] \\
\hline & $\begin{array}{l}\text { Myelodysplastic } \\
\text { syndrome (MDS) }\end{array}$ & Decreased MNDA protein expression in marrow samples from patients with MDS & [81] \\
\hline & Sepsis & $\begin{array}{l}\text { Cytoplasmic accumulation of and cleavage of MNDA in neutrophils is impaired resulting in less } \\
\text { neutrophil apoptosis }\end{array}$ & {$[12]$} \\
\hline & Sjögren's syndrome (SjS) & Elevated MNDA mRNA in patients with primary SjS & [82] \\
\hline \multirow[t]{3}{*}{ PYHIN1/IFIX } & Asthma & SNPs in the PYHIN1 locus linked to susceptibility for individuals of African descent & {$[60]$} \\
\hline & Breast cancer & Downregulated PYHIN1 mRNA expression in breast cancer tissues & [6] \\
\hline & $\begin{array}{l}\text { Systolic blood } \\
\text { pressure (SBP) }\end{array}$ & SNP near PYHIN1 with a suggestive association with SBP in African Americans & [83] \\
\hline
\end{tabular}




\section{Therapeutic approaches to targeting PYHIN proteins}

While it is clear that the PYHIN family plays a central role in innate immunity, the evidence to date for the involvement of the PYHINs in disease mainly comes from altered expression patterns in disease, the presence of auto-antibodies against these proteins in sera of patients and single nucleotide polymorphisms in the genomic regions encoding the PYHINs (Table 1 ).

Since aberrant and excessive DNA sensing contributes to autoimmune and inflammatory diseases [69] the involvement of the ALRs AIM2 and IFI16 at an early point in innate immune activation with regard to their role in DNA sensing, positions them at an ideal point for therapeutic targeting. Caspase- 1 , which is activated following HIV DNA recognition by IFI16 [51] has been targeted using a caspase- 1 inhibitor that is safe for use in humans. This inhibitor has been shown to block caspase- 1 cleavage, IL-1 $\beta$ secretion and $\mathrm{CD} 4^{+} \mathrm{T}$ cell death [70], demonstrating that inhibiting IFI16 induced signalling in HIV patients has great therapeutic promise.

However, as discussed earlier the DNA binding capabilities of the human PYHINs are not solely utilised in viral detection, as the human PYHINs can also act as transcriptional regulators. In addition, most ALRs bind dsDNA in a non-sequence specific manner, so the use of synthetic oligodeoxynucleotides (ODN) which can bind DNA sensors in a manner that is competitive with immune stimulatory DNA must be highly specific to avoid affecting the functions of other ALRs. For example ODN-A151 has been shown to inhibit dsDNA induced type I IFN, TNF $\alpha$, ISG induction and caspase 1 dependent IL-1 $\beta$ and IL-18 maturation by binding to AIM2. However, biotinylated ODN-A151 also immunoprecipitated with IFI16, further suggesting that multiple dsDNA induced signalling events are likely to be effected by ODN-A151 [71]. LL-37 is a human cathelicidin antimicrobial peptide that forms a complex with extracellular self-DNA, sequestering it and inhibiting the ability of DNA to induce IL-1 $\beta$ production via the AIM2 inflammasome. However, LL-37 promotes cellular uptake of self-DNA and while LL37/self-DNA complexes do not appear to activate AIM2, they do initiate inflammatory cascades via TLR9 [63]. This highlights the difficulty in targeting a molecule which can exert effects via multiple PRRs.

Alternatively, targeting a more specific association such as those initiated directly after DNA:ALR binding for instance PYD:PYD interactions may be more promising. It is becoming apparent that oligomerisation is a unifying mechanism critical for downstream signal activation $[29,43,47,58]$. DsDNA binding to IFI16 and AIM2 induces the polymerisation of IFI16 PYD domains, or the polymerisation of AIM2PYD and ASCPYD respectively. Targeting these key associations, which determine the stability of the signal activation complexes, could encourage their disruption thereby alleviating autoimmune disorders. Alternatively, complex stability could be improved, resulting in enhanced immunity. The elucidation of crystal structures for IFI16 PYD:PYD associations AIM2PYD:ASCPYD would be extremely advantageous in this regard.

Viral inhibitors of the PYHINs such as pUL83 may also prove very informative. Understanding how this and other viral inhibitors work may aid the design of specific peptide inhibitors of human PYHINs. This approach has proved successful in other contexts, for example a peptide derived from the vaccinia virus protein A46 (known as VIPER) is capable of inhibiting TLR induced signalling by interfering with downstream adapter protein associations which are required for signal activation [72]. In the context of DNA sensing, PYHINs operate within the cell therefore intracellular delivery of therapeutics will be a crucial step to overcome. However, targeting circulating IFI16 for example may be another promising way to treat autoimmune and inflammatory disorders.
Another challenge in the development of therapeutics targeting proteins of the PYHIN family will be to usefully interpret data from mouse models. Given the complexity of the PYHIN family in mouse, which have at least 13 members [7,73] it may be difficult to determine the correlation if any, with data from humans studies. Although human IFI16 and mouse p204 have been shown to function similarly, and have a similar domain structure containing a PYRIN domain and two HIN domains, human and mouse AIM2 are the only direct orthologues identified between these species based on the phylogenetic analyses of both HIN and pyrin domains [7,73]. While human and mouse PYHINs such as IFI16 and p204 appear to be functional orthologues, it will nonetheless be important to bear in mind the many differences between these two systems. The regulation of the PYHINs may also differ between the mouse and human systems, and further complicate the interpretation of data. For example, POP3, which negatively regulates AIM2 and IFI16, is absent in mouse [3].

\section{Concluding remarks}

In recent years significant progress has been made regarding the PYHIN proteins, particularly considering the roles of ALRs in DNA sensing. While the IFN inducible gene cluster has been known for many years, the very recent discovery of a novel protein encoded in this region (POP3) emphasises that there may be numerous exciting discoveries still to be made. For example, the existence of non-coding RNAs in this region with potentially novel regulatory roles has yet to be investigated. Furthermore roles for MNDA and PYHIN1 in innate immunity remain to be determined. In the coming years we hope to gain a deeper understanding of the diverse roles of these proteins. In addition, understanding how exactly their various functions are regulated both by the host and by viruses will be extremely informative. Interestingly, the PYHINs are not the only protein family with dual roles as innate immune sensors and transcriptional regulators. For example, the NLRs NLRC5 and NLRP12 have additional roles acting as transcriptional regulators of MHC class I, and the associated genes $\beta 2 \mathrm{M}$, TAP1, and LMP2 [74,75]. Future work will likely reveal further gene regulatory roles for the PYHINs both as nucleic acid sensors and as downstream components of signal transduction pathways. In addition, investigation into the differential molecular and cellular events involved in ALR-stimulated STING or inflammasome activation will greatly advance our understanding of downstream pathway activation and reveal novel therapeutic opportunities. While great progress has undoubtedly been made in recent years with regard to the roles of human PYHINs and their mechanisms of action, we are some way from reaping the benefits of targeted PYHIN control by therapeutic means. Further research on these proteins will certainly yield important information that we require for a more complete understanding of their roles, particularly in disease. In turn this will greatly aid the development of therapeutics targeting PYHIN proteins.

\section{Acknowledgements}

Work on PYHINs in the authors' lab is funded by grants from Science Foundation Ireland (11/PI/1056) and the National Institutes of Health (AI093752).

\section{References}

[1] Kawai T, Akira S. The roles of TLRs, RLRs and NLRs in pathogen recognition. Int Immunol 2009;21:317-37.

[2] Unterholzner L, Keating SE, Baran M, Horan KA, Jensen SB, Sharma S, et al. IFI16 is an innate immune sensor for intracellular DNA. Nat Immunol 2010;11:997-1004

[3] Khare S, Ratsimandresy RA, de Almeida L, Cuda CM, Rellick SL, Misharin AV, et al. The PYRIN domain-only protein POP3 inhibits ALR inflammasomes and 
regulates responses to infection with DNA viruses. Nat Immunol 2014;15: 343-53.

[4] Briggs JA, Burrus GR, Stickney BD, Briggs RC. Cloning and expression of the human myeloid cell nuclear differentiation antigen: regulation by interferon alpha. J Cell Biochem 1992;49:82-92.

[5] Veeranki S, Duan X, Panchanathan R, Liu H, Choubey D. IFI16 protein mediates the anti-inflammatory actions of the type-I interferons through suppression of activation of caspase-1 by inflammasomes. PLoS One 2011;6:e27040.

[6] Ding Y, Wang L, Su LK, Frey JA, Shao R, Hunt KK, et al. Antitumor activity of IFIX, a novel interferon-inducible $\mathrm{HIN}-200$ gene, in breast cancer. Oncogene 2004;23:4556-66.

[7] Cridland JA, Curley EZ, Wykes MN, Schroder K, Sweet MJ, Roberts TL, et al. The mammalian PYHIN gene family: phylogeny, evolution and expression. BMC Evol Biol 2012;12:140.

[8] Ludlow LE, Johnstone RW, Clarke CJ. The HIN-200 family: more than interferon-inducible genes. Exp Cell Res 2005;308:1-17.

[9] Hornung V, Ablasser A, Charrel-Dennis M, Bauernfeind F, Horvath G, Caffrey DR, et al. AIM2 recognizes cytosolic dsDNA and forms a caspase-1-activating inflammasome with ASC. Nature 2009;458:514-8.

[10] Li T, Diner BA, Chen J, Cristea IM. Acetylation modulates cellular distribution and DNA sensing ability of interferon-inducible protein IFI16. Proc Natl Acad Sci U S A 2012;109:10558-63.

[11] Kerur N, Veettil MV, Sharma-Walia N, Bottero V, Sadagopan S, Otageri P, et al. IFI16 acts as a nuclear pathogen sensor to induce the inflammasome in response to Kaposi Sarcoma-associated herpesvirus infection. Cell Host Microbe 2011:9:363-75.

[12] Fotouhi-Ardakani N, Kebir DE, Pierre-Charles N, Wang L, Ahern SP, Filep JG, et al. Role for myeloid nuclear differentiation antigen in the regulation of neutrophil apoptosis during sepsis. Am J Respir Crit Care Med 2010;182: $341-50$.

[13] Goldberger A, Hnilica LS, Casey SB, Briggs RC. Properties of a nuclear protein marker of human myeloid cell differentiation. J Biol Chem 1986;261:4726-31.

[14] Xie J, Briggs JA, Olson MO, Sipos K, Briggs RC. Human myeloid cell nuclea differentiation antigen binds specifically to nucleolin. J Cell Biochem 1995;59:529-36.

[15] Xie J, Briggs JA, Briggs RC. MNDA dimerizes through a complex motif involving an N-terminal basic region. FEBS Lett 1997:408:151-5.

[16] Xie J, Briggs JA, Briggs RC. Human hematopoietic cell specific nuclear protein MNDA interacts with the multifunctional transcription factor YY1 and stimulates YY1 DNA binding. I Cell Biochem 1998;70:489-506.

[17] Doggett KL, Briggs JA, Linton MF, Fazio S, Head DR, Xie J, et al. Retroviral mediated expression of the human myeloid nuclear antigen in a null cell line upregulates Dlk1 expression. J Cell Biochem 2002;86:56-66.

[18] Suzuki T, Nakano-Ikegaya M, Yabukami-Okuda H, de Hoon M, Severin J, SagaHatano S, et al. Reconstruction of monocyte transcriptional regulatory network accompanies monocytic functions in human fibroblasts. PLoS One 2012; 7:e33474

[19] Li H, Wang ZX, Wu JW. Purification, characterization and docking studies of the HIN domain of human myeloid nuclear differentiation antigen (MNDA) Biotechnol Lett 2014;36:899-905.

[20] Johnstone RW, Kershaw MH, Trapani JA. Isotypic variants of the interferoninducible transcriptional repressor IFI 16 arise through differential mRNA splicing. Biochemistry 1998;37:11924-31.

[21] Johnstone RW, Wei W, Greenway A, Trapani JA. Functional interaction between p53 and the interferon-inducible nucleoprotein IFI 16. Oncogene 2000;19:6033-42.

[22] Egistelli L, Chichiarelli S, Gaucci E, Eufemi M, Schinina ME, Giorgi A, et al. IFI16 and NM23 bind to a common DNA fragment both in the P53 and the cMYC gene promoters. J Cell Biochem 2009;106:666-72.

[23] Aglipay JA, Lee SW, Okada S, Fujiuchi N, Ohtsuka T, Kwak JC, et al. A member of the Pyrin family, IFI16, is a novel BRCA1-associated protein involved in the p53-mediated apoptosis pathway. Oncogene 2003;22:8931-8.

[24] Xin H, Curry J, Johnstone RW, Nickoloff BJ, Choubey D. Role of IFI 16, a member of the interferon-inducible p200-protein family, in prostate epithelial cellular senescence. Oncogene 2003;22:4831-40.

[25] Gariano GR, Dell'Oste V, Bronzini M, Gatti D, Luganini A, De Andrea M, et al. The intracellular DNA sensor IFI16 gene acts as restriction factor for human cytomegalovirus replication. PLoS Pathog 2012;8:e1002498.

[26] Thompson MR, Sharma S, Atianand M, Jensen SB, Carpenter S, Knipe DM, et al. Interferon Gamma Inducible protein (IFI)16 transcriptionally regulates type interferons and other interferon stimulated genes and controls the interferon response to both DNA and RNA viruses. J Biol Chem 2014.

[27] DeYoung KL, Ray ME, Su YA, Anzick SL, Johnstone RW, Trapani JA, et al. Cloning a novel member of the human interferon-inducible gene family associated with control of tumorigenicity in a model of human melanoma. Oncogene 1997:15:453-7.

[28] Harada M, Li YF, El-Gamil M, Ohnmacht GA, Rosenberg SA, Robbins PF. Melanoma-Reactive CD8+ T cells recognize a novel tumor antigen expressed in a wide variety of tumor types. J Immunother 2001;24:323-33.

[29] Li T, Chen J, Cristea IM. Human cytomegalovirus tegument protein pUL83 inhibits IFI16-mediated DNA sensing for immune evasion. Cell Host Microbe $2013 ; 14: 591-9$.

[30] Cresswell KS, Clarke CJ, Jackson JT, Darcy PK, Trapani JA, Johnstone RW. Biochemical and growth regulatory activities of the HIN-200 family member and putative tumor suppressor protein, AIM2. Biochem Biophys Res Commun 2005;326:417-24.
[31] Ding Y, Lee JF, Lu H, Lee MH, Yan DH. Interferon-inducible protein IFIXalpha1 functions as a negative regulator of HDM2. Mol Cell Biol 2006:26:1979-96.

[32] Yamaguchi H, Ding Y, Lee JF, Zhang M, Pal A, Bornmann W, et al. Interferoninducible protein IFIXalpha inhibits cell invasion by upregulating the metastasis suppressor maspin. Mol Carcinogen 2008;47:739-43.

[33] Hemmi H, Takeuchi O, Kawai T, Kaisho T, Sato S, Sanjo H, et al. A Toll-like receptor recognizes bacterial DNA. Nature 2000;408:740-5.

[34] Hayashi T, Nishitsuji H, Takamori A, Hasegawa A, Masuda T, Kannagi M. DNAdependent activator of IFN-regulatory factors enhances the transcription of HIV1 through NF-kappaB. Microbes Infection/Institut Pasteur 2010;12:937-47.

[35] Unterholzner L. The interferon response to intracellular DNA: why so many receptors. Immunobiology 2013;218:1312-21.

[36] Bowie AG. Rad50 and CARD9, missing links in cytosolic DNA-stimulated inflammation. Nat Immunol 2014;15:534-6.

[37] Albrecht M, Choubey D, Lengauer T. The HIN domain of IFI-200 proteins consists of two OB folds. Biochem Biophys Res Commun 2005:327:679-87.

[38] Rathinam VA, Jiang Z, Waggoner SN, Sharma S, Cole LE, Waggoner L, et al. The AIM2 inflammasome is essential for host defense against cytosolic bacteria and DNA viruses. Nat Immunol 2010;11:395-402.

[39] Saiga H, Kitada S, Shimada Y, Kamiyama N, Okuyama M, Makino M, et al. Critical role of AIM2 in Mycobacterium tuberculosis infection. Int Immunol 2012;24:637-44

[40] Manzanillo PS, Shiloh MU, Portnoy DA, Cox JS. Mycobacterium tuberculosis activates the DNA-dependent cytosolic surveillance pathway within macrophages. Cell Host Microbe 2012;11:469-80.

[41] Jin T, Perry A, Jiang J, Smith P, Curry JA, Unterholzner L, et al. Structures of the HIN domain:DNA complexes reveal ligand binding and activation mechanisms of the AIM2 inflammasome and IFI16 receptor. Immunity 2012;36:561-71.

[42] Jin T, Perry A, Smith P, Jiang J, Xiao TS. Structure of the absent in melanoma 2 (AIM2) pyrin domain provides insights into the mechanisms of AIM2 autoinhibition and inflammasome assembly. J Biol Chem 2013;288:13225-35.

[43] Cai X, Chen J, Xu H, Liu S, Jiang QX, Halfmann R, et al. Prion-like polymerization underlies signal transduction in antiviral immune defense and inflammasome activation. Cell 2014;156:1207-22.

[44] Ansari MA, Singh VV, Dutta S, Veettil MV, Dutta D, Chikoti L, et al. Constitutive interferon-inducible protein 16-inflammasome activation during Epstein-Barr virus latency I, II, and III in B and epithelial cells. J Virol 2013;87:8606-23.

[45] Johnson KE, Chikoti L, Chandran B. Herpes simplex virus 1 infection induces activation and subsequent inhibition of the IFI16 and NLRP3 inflammasomes. J Virol 2013:87:5005-18.

[46] Jakobsen MR, Bak RO, Andersen A, Berg RK, Jensen SB, Tengchuan J, et al. IFI16 senses DNA forms of the lentiviral replication cycle and controls HIV-1 replication. Proc Natl Acad Sci U S A 2013;110:E4571-80.

[47] Morrone SR, Wang T, Constantoulakis LM, Hooy RM, Delannoy MJ, Sohn J. Cooperative assembly of IFI16 filaments on dsDNA provides insights into host defense strategy. Proc Natl Acad Sci U S A 2014;111:E62-71.

[48] Horan KA, Hansen K, Jakobsen MR, Holm CK, Soby S, Unterholzner L, et al. Proteasomal degradation of herpes simplex virus capsids in macrophages releases DNA to the cytosol for recognition by DNA sensors. J Immunol 2013;190:2311-9.

[49] Dell'Oste V, Gatti D, Gugliesi F, De Andrea M, Bawadekar M, Lo Cigno I, et al. Innate nuclear sensor IFI16 translocates into the cytoplasm during the early stage of in vitro human cytomegalovirus infection and is entrapped in the egressing virions during the late stage. J Virol 2014;88:6970-82.

[50] Hansen K, Prabakaran T, Laustsen A, Jorgensen SE, Rahbaek SH, Jensen SB, et al. Listeria monocytogenes induces IFNbeta expression through an IFI16-, cGASand STING-dependent pathway. EMBO J 2014.

[51] Monroe KM, Yang Z, Johnson JR, Geng X, Doitsh G, Krogan NJ, et al. IFI16 DNA sensor is required for death of lymphoid CD4T cells abortively infected with HIV. Science 2014;343:428-32.

[52] Roberts TL, Idris A, Dunn JA, Kelly GM, Burnton CM, Hodgson S, et al. HIN-200 proteins regulate caspase activation in response to foreign cytoplasmic DNA. Science 2009;323:1057-60.

[53] Yin Q, Sester DP, Tian Y, Hsiao YS, Lu A, Cridland JA, et al. Molecular mechanism for p202-mediated specific inhibition of AIM2 inflammasome activation. Cell Rep 2013;4:327-39

[54] Choubey D, Duan X, Dickerson E, Ponomareva L, Panchanathan R, Shen H, et al. Interferon-inducible p200-family proteins as novel sensors of cytoplasmic DNA: role in inflammation and autoimmunity. J Interferon Cytokine Res 2010;30:371-80

[55] Orzalli MH, DeLuca NA, Knipe DM. Nuclear IFI16 induction of IRF-3 signaling during herpesviral infection and degradation of IFI16 by the viral ICP0 protein. Proc Natl Acad Sci U S A 2012;109:E3008-17.

[56] Cuchet-Lourenco D, Anderson G, Sloan E, Orr A, Everett RD. The viral ubiquitin ligase ICP0 is neither sufficient nor necessary for degradation of the cellular DNA sensor IFI16 during herpes simplex virus 1 infection. J Virol 2013;87:13422-32.

[57] Fukushi M, Higuchi M, Oie M, Tetsuka T, Kasolo F, Ichiyama K, et al. Latencyassociated nuclear antigen of Kaposi's sarcoma-associated herpesvirus interacts with human myeloid cell nuclear differentiation antigen induced by interferon alpha. Virus Genes 2003;27:237-47

[58] Lu A, Magupalli VG, Ruan J, Yin Q, Atianand MK, Vos MR, et al. Unified polymerization mechanism for the assembly of ASC-dependent inflammasomes. Cell 2014;156:1193-206.

[59] Choubey D, Panchanathan R. Interferon-inducible Ifi200-family genes in systemic lupus erythematosus. Immunol Lett 2008;119:32-41. 
[60] Torgerson DG, Ampleford EJ, Chiu GY, Gauderman WJ, Gignoux CR, Graves PE, et al. Meta-analysis of genome-wide association studies of asthma in ethnically diverse North American populations. Nat Genet 2011;43: 887-92.

[61] Ponomareva L, Liu H, Duan X, Dickerson E, Shen H, Panchanathan R, et al. AIM2, an IFN-inducible cytosolic DNA sensor, in the development of benign prostate hyperplasia and prostate cancer. Mol Cancer Res: MCR 2013;11:1193-202.

[62] Dihlmann S, Tao S, Echterdiek F, Herpel E, Jansen L, Chang-Claude J, et al. Lack of absent in melanoma 2 (AIM2) expression in tumor cells is closely associated with poor survival in colorectal cancer patients. Int J Cancer 2014.

[63] Dombrowski Y, Peric M, Koglin S, Kammerbauer C, Goss C, Anz D, et al. Cytosolic DNA triggers inflammasome activation in keratinocytes in psoriatic lesions. Sci Translational Med 2011;3:82ra38.

[64] Caneparo V, Cena T, De Andrea M, Dell'oste V, Stratta P, Quaglia M, et al. AntiIFI16 antibodies and their relation to disease characteristics in systemic lupus erythematosus. Lupus 2013;22:607-13.

[65] Costa S, Mondini M, Caneparo V, Afeltra A, Airo P, Bellisai F, et al. Detection of anti-IFI16 antibodies by ELISA: clinical and serological associations in systemic sclerosis. Rheumatology 2011;50:674-81.

[66] Gugliesi F, Bawadekar M, De Andrea M, Dell'Oste V, Caneparo V, Tincani A, et al. Nuclear DNA sensor IFI16 as circulating protein in autoimmune diseases is a signal of damage that impairs endothelial cells through high-affinity membrane binding. PLoS One 2013;8:e63045.

[67] Uchida K, Akita Y, Matsuo K, Fujiwara S, Nakagawa A, Kazaoka Y, et al. Identification of specific autoantigens in Sjogren's syndrome by SEREX. Immunology 2005;116:53-63.

[68] Nissen SK, Hojen JF, Andersen KL, Kofod-Olsen E, Berg RK, Paludan SR, et al. Innate DNA sensing is impaired in HIV patients and IFI16 expression correlates with chronic immune activation. Clin Exp Immunol 2014;177:295-309.

[69] Paludan SR, Bowie AG. Immune sensing of DNA. Immunity 2013;38:870-80.

[70] Doitsh G, Galloway NL, Geng X, Yang Z, Monroe KM, Zepeda O, et al. Cell death by pyroptosis drives CD4 T-cell depletion in HIV-1 infection. Nature 2014:505:509-14.

[71] Kaminski JJ, Schattgen SA, Tzeng TC, Bode C, Klinman DM, Fitzgerald KA. Synthetic oligodeoxynucleotides containing suppressive TTAGGG motifs inhibit AIM2 inflammasome activation. J Immunol 2013;191:3876-83.

[72] Lysakova-Devine T, Keogh B, Harrington B, Nagpal K, Halle A, Golenbock DT, et al. Viral inhibitory peptide of TLR4, a peptide derived from vaccinia protein
A46, specifically inhibits TLR4 by directly targeting MyD88 adaptor-like and TRIF-related adaptor molecule. J Immunol 2010;185:4261-71.

[73] Brunette RL, Young JM, Whitley DG, Brodsky IE, Malik HS, Stetson DB. Extensive evolutionary and functional diversity among mammalian AIM2-like receptors. J Exp Med 2012;209:1969-83.

[74] Meissner TB, Li A, Biswas A, Lee KH, Liu YJ, Bayir E, et al. NLR family member NLRC5 is a transcriptional regulator of MHC class I genes. Proc Natl Acad Sci U S A 2010;107:13794-99.

[75] Williams KL, Taxman DJ, Linhoff MW, Reed W, Ting JP. Cutting edge: Monarch1: a pyrin/nucleotide-binding domain/leucine-rich repeat protein that controls classical and nonclassical MHC class I genes. J Immunol 2003;170:5354-8.

[76] Dihlmann S, Erhart P, Mehrabi A, Nickkholgh A, Lasitschka F, Bockler D, et al. Increased expression and activation of Absent in Melanoma 2 inflammasome components in lymphocytic infiltrates of Abdominal Aortic Aneurysms. Mol Med 2014.

[77] Zhen J, Zhang L, Pan J, Ma S, Yu X, Li X, et al. AIM2 mediates inflammation-associated renal damage in hepatitis B virus-associated glomerulonephritis by regulating caspase-1, IL-1beta, and IL-18. Mediators Inflammation 2014;2014:190860.

[78] Javierre BM, Fernandez AF, Richter J, Al-Shahrour F, Martin-Subero JI, RodriguezUbreva J, et al. Changes in the pattern of DNA methylation associate with twin discordance in systemic lupus erythematosus. Genome Res 2010;20:170-9.

[79] Mondini M, Vidali M, De Andrea M, Azzimonti B, Airo P, D‘Ambrosio R, et al. A novel autoantigen to differentiate limited cutaneous systemic sclerosis from diffuse cutaneous systemic sclerosis: the interferon-inducible gene IFI16. Arthritis Rheumatism 2006;54:3939-44.

[80] Metcalf RA, Monabati A, Vyas M, Roncador G, Gualco G, Bacchi CE, et al. Myeloid cell nuclear differentiation antigen is expressed in a subset of marginal zone lymphomas and is useful in the differential diagnosis with follicular lymphoma. Hum Pathol 2014.

[81] McClintock-Treep SA, Briggs RC, Shults KE, Flye-Blakemore LA, Mosse CA, Jagasia $\mathrm{MH}$, et al. Quantitative assessment of myeloid nuclear differentiation antigen distinguishes myelodysplastic syndrome from normal bone marrow. Am J Clin Pathol 2011;135:380-5.

[82] Hu S, Gao K, Pollard R, Arellano-Garcia M, Zhou H, Zhang L, et al. Preclinical validation of salivary biomarkers for primary Sjogren's syndrome. Arthritis Care Res 2010;62:1633-8.

[83] de Las Fuentes L, Sung YJ, Schwander KL, Kalathiveetil S, Hunt SC, Arnett DK, et al. The role of SNP-loop diuretic interactions in hypertension across ethnic groups in HyperGEN. Frontiers Genet 2013;4:304. 